\title{
Induced Fuzzy K-Ideal and Its Level K-Ideals on a HX Ring
}

\author{
R. Muthuraj ${ }^{1}$, N.Ramila Gandhi ${ }^{2}$ \\ ${ }^{1} P G \&$ Reseach Department of Mathematics, H.H.The Rajah's College, Pudukkottai-622 001, Tamilnadu, India. \\ ${ }^{2}$ Department of Mathematics, PSNA College of Engineering and Technology, Dindigul-624 622, Tamilnadu, India.
}

\begin{abstract}
In this paper, we define a new algebraic structure of an induced fuzzy k-ideal of a HX ring and some related properties are investigated. The purpose of this study is to implement the fuzzy set theory and ring theory in fuzzy HX k-ideals of a HX ring. We also establish the relation between fuzzy HX ideals and fuzzy HX $k$-ideals of a HX ring . Characterizations of level subsets of a fuzzy HX k-ideal of a HX ring are given. We also discussed the relation between a given fuzzy $H X$-ideals of a $H X$ ring and its level $H X$ k-ideals and investigate the conditions under which a given $H X$ ring has a properly inclusive chain of $H X$ k-ideals. In particular, we formulate how to structure a fuzzy HX k-ideal of a HX ring by a given chain of HX k-ideals.
\end{abstract}

Keywords: fuzzy ideal, fuzzy k-ideal, fuzzy HX ring, fuzzy HX ideal, fuzzy HX k-ideal, level HX k-ideals.

\section{Introduction}

In 1965, Zadeh [16] introduced the concept of fuzzy subset $\mu$ of a set $\mathrm{X}$ as a function from $\mathrm{X}$ into the closed unit interval $[0,1]$ and studied their properties. Fuzzy set theory is a useful tool to describe situations in which the data or imprecise or vague and it is applied to logic, set theory, group theory, ring theory, real analysis, measure theory etc. In 1967, Rosenfeld [14] defined the idea of fuzzy subgroups and gave some of its properties. Li Hong Xing [9] introduced the concept of HX group. In 1982 Wang-jin Liu[10] introduced the concept of fuzzy ring and fuzzy ideal. Liu defined the fuzzy ideals of a ring and discussed the operations on fuzzy ideals. T.K.Dutta and B.K.Biswas [5] studied fuzzy ideals and fuzzy prime k-ideals of semi rings.The class of $\mathrm{k}$-fuzzy semi rings, where $\mathrm{k}$ denotes a subset of the power set of $\mathrm{R}$ which is closed under the operations min, + , or max has many interesting applications. Min-max-plus computations are used in several areas. The notion of k-ideals was applied to construct quotient rings. With the successful upgrade of algebraic structure of group many researchers considered the algebraic structure of some other algebraic systems in which ring was considered as first. In 1988, Professor Li Hong Xing [11] proposed the concept of HX ring and derived some of its properties, then Professor Zhong [1,2] gave the structures of HX ring on a class of ring. In this paper we define a new algebraic structure of an induced fuzzy HX ideal, induced fuzzy HX k-ideal of a HX ring and investigate some related properties.

\section{Preliminaries}

In this section, we site the fundamental definitions that will be used in the sequel. Throughout this paper, $\mathrm{R}=(\mathrm{R},+, \cdot)$ is a Ring, e is the additive identity element of $\mathrm{R}$ and $\mathrm{xy}$, we mean $\mathrm{x} . \mathrm{y}$

\subsection{Definition [3]}

A non-empty set $\mathrm{R}$ together with two binary operations ' + ' and '.' is said to be a ring if the following conditions are satisfied. For all a,b,c $\in \mathrm{R}$,
i. $\quad(\mathrm{R},+)$ is an abelian group,
ii. $\quad(\mathrm{R}, \cdot)$ is a semi group,
iii. $\quad a(b+c)=a b+a c$ and $(a+b) c=a c+b c$

\subsection{Definition [15]}

A non-empty subset $I$ of a ring $R$ is said to be a left ideal of $R$ if $x, y \in I$ and $r \in R$ imply that $x+y \in I$ and $r x \in I$. A non-empty subset $I$ of a ring $R$ is said to be a right ideal of $R$ if $x, y \in I$ and $r \in R$ imply that $\mathrm{x}+\mathrm{y} \in \mathrm{I}$ and $\mathrm{xr} \in \mathrm{I}$. A non-empty subset $\mathrm{I}$ of a ring $\mathrm{R}$ is said to be an ideal or a two sided ideal of $\mathrm{R}$ if $\mathrm{I}$ is both left and right ideal of $\mathrm{R}$.

\subsection{Definition [15]}

A left ideal $I$ of a ring $R$ is said to be a left k-ideal of $R$ if $x \in I$ and $r \in R$ and $x+r \in I$ or $r+x \in I$ then $r \in I$. A right ideal $I$ of a ring $R$ is said to be a right $k$-ideal of $R$ if $x \in I$ and $r \in R$ and $x+r \in I$ or $r+x \in I$ then $r \in I$. An ideal $I$ of a ring $R$ is said to be a k-ideal of $R$ if $I$ is both left and right k-ideal of $R$. 


\subsection{Definition [4]}

A fuzzy subset $\mu$ in a ring $\mathrm{R}$ is called fuzzy ideal of $\mathrm{R}$ if the following conditions are satisfied. For all $\mathrm{x}, \mathrm{y} \in \mathrm{R}$,

$$
\begin{array}{lll}
\text { i. } & \mu(\mathrm{x}-\mathrm{y}) & \geq \min \{\mu(\mathrm{x}), \mu(\mathrm{y})\}, \\
\text { ii. } & \mu(\mathrm{xy}) & \geq \mu(\mathrm{y}), \\
\text { iii. } & \mu(\mathrm{xy}) & \geq \mu(\mathrm{x}) .
\end{array}
$$

If (i) and (ii) are satisfied then it is called fuzzy left ideal of R. If (i) and (iii) are satisfied then it is called fuzzy right ideal of $\mathrm{R}$.

\subsection{Definition [15]} $\mathrm{x}, \mathrm{y}$ in $\mathrm{R}$.

A fuzzy ideal $\mu$ of a ring $\mathrm{R}$ is said to be a fuzzy $\mathrm{k}$-ideal of $\mathrm{R}$ if $\mu(\mathrm{x}) \geq \min \{\mu(\mathrm{x}+\mathrm{y}), \mu(\mathrm{y})\}$ for all

\subsection{Definition [3]}

Let $\mu, \lambda$ be any two fuzzy subsets of a set $\mathrm{X}$. A fuzzy subset $\mu \cap \lambda$ is defined as $(\mu \cap \lambda)(\mathrm{x})=\min \{\mu(\mathrm{x}), \lambda(\mathrm{x})\}$ for all $\mathrm{x} \in \mathrm{X}$.

\section{Properties Of An Induced Fuzzy Ideal (Fuzzy HX K-Ideal) Of A HX Ring}

\subsection{Definition [1]}

Let $\mathrm{R}$ be a ring. In $2^{\mathrm{R}}-\{\phi\}$, a non-empty set $\vartheta \subset 2^{\mathrm{R}}-\{\phi\}$ with two binary operation ' + ' and '.' is said to be a HX ring on $\mathrm{R}$ if $\vartheta$ is a ring with respect to the algebraic operation defined by

i. $\quad A+B=\{a+b / a \in A$ and $b \in B\}$, which its null element is denoted by $Q$, and the negative element of $A$ is denoted by $-A$.

ii. $\quad \mathrm{AB}=\{\mathrm{ab} / \mathrm{a} \in \mathrm{A}$ and $\mathrm{b} \in \mathrm{B}\}$,

iii. $\quad \mathrm{A}(\mathrm{B}+\mathrm{C})=\mathrm{AB}+\mathrm{AC}$ and $(\mathrm{B}+\mathrm{C}) \mathrm{A}=\mathrm{BA}+\mathrm{CA}$.

\subsection{Definition}

Let $R$ be a ring. Let $\mu$ be a fuzzy ring defined on R. Let $\vartheta \subset 2^{R}-\{\phi\}$ be a HX ring. A fuzzy subset $\lambda^{\mu}$ of $\vartheta$ is called a fuzzy HX ring on $\vartheta$ or a fuzzy ring induced by $\mu$ if the following conditions are satisfied. For all $\mathrm{A}, \mathrm{B} \in \vartheta$,

$$
\begin{array}{ll}
\text { i. } & \lambda^{\mu}(\mathrm{A}-\mathrm{B}) \geq \min \left\{\lambda^{\mu}(\mathrm{A}), \lambda^{\mu}(\mathrm{B})\right\}, \\
\text { ii. } & \lambda^{\mu}(\mathrm{A} \mathrm{B}) \geq \min \left\{\lambda^{\mu}(\mathrm{A}), \lambda^{\mu}(\mathrm{B})\right\}
\end{array}
$$

where $\lambda^{\mu}(\mathrm{A})=\max \{\mu(\mathrm{x}) /$ for all $\mathrm{x} \in \mathrm{A} \subseteq \mathrm{R}\}$.

\subsection{Definition}

Let $\mu$ be a fuzzy ideal defined on R. Let $\vartheta \subset 2^{R}-\{\phi\}$ be a HX ring. A fuzzy subset $\lambda^{\mu}$ of $\vartheta$ is said to be a fuzzy HX ideal or fuzzy ideal induced by $\mu$ on $\vartheta$ if the following conditions are satisfied. For all A,B $\in \vartheta$,

$$
\begin{array}{lll}
\text { i. } & \lambda^{\mu}(\mathrm{A}-\mathrm{B}) & \geq \min \left\{\lambda^{\mu}(\mathrm{A}), \lambda^{\mu}(\mathrm{B})\right\}, \\
\text { ii. } & \lambda^{\mu}(\mathrm{AB}) & \geq \lambda^{\mu}(\mathrm{B}), \\
\text { iii. } & \lambda^{\mu}(\mathrm{AB}) & \geq \lambda^{\mu}(\mathrm{A})
\end{array}
$$

where $\lambda^{\mu}(\mathrm{A})=\max \{\mu(\mathrm{x}) /$ for all $\mathrm{x} \in \mathrm{A} \subseteq \mathrm{R}\}$

If (i) and (ii) are satisfied then it is fuzzy HX left ideal of $\vartheta$. If (i) and (iii) are satisfied then it is called fuzzy HX right ideal of $\vartheta$.

\subsection{Definition}

Let $\mu$ be a fuzzy ideal defined on R. Let $\vartheta \subset 2^{R}-\{\phi\}$ be a HX ring. A fuzzy subset $\lambda^{\mu}$ of $\vartheta$ is said to be a fuzzy HX k-ideal or a fuzzy k ideal induced by $\mu$ on $\vartheta$ if $\lambda^{\mu}(A) \geq \min \left\{\lambda^{\mu}(A+B), \lambda^{\mu}(B)\right\}$,for all $A, B \in \vartheta$.

\subsection{Theorem}

Proof

If $\mu$ is a fuzzy ideal of $\mathrm{R}$ then the induced fuzzy subset $\lambda^{\mu}$ is a fuzzy $\mathrm{HX}$ ideal on $\vartheta$.

Let $\mu$ be a fuzzy ideal of R.

i. $\min \left\{\lambda^{\mu}(\mathrm{A}), \lambda^{\mu}(\mathrm{B})\right\} \quad=\min \{\max \{\mu(\mathrm{x}) /$ for all $\mathrm{x} \in \mathrm{A} \subseteq \mathrm{R}\}, \max \{\mu(\mathrm{y}) /$ for all $\mathrm{y} \in \mathrm{B} \subseteq \mathrm{R}\}\}$

$=\min \left\{\mu\left(\mathrm{x}_{0}\right), \mu\left(\mathrm{y}_{0}\right)\right\}$

$\leq \mu\left(x_{0}-y_{0}\right)$, since $\mu$ is a fuzzy ideal of $R$

$\leq \max \{\mu(\mathrm{x}-\mathrm{y}) /$ for all $\mathrm{x}-\mathrm{y} \in \mathrm{A}-\mathrm{B} \subseteq \mathrm{R}\}$

$\leq \lambda^{\mu}(\mathrm{A}-\mathrm{B})$ 


$$
\lambda^{\mu}(\mathrm{A}-\mathrm{B}) \quad \geq \min \left\{\lambda^{\mu}(\mathrm{A}), \lambda^{\mu}(\mathrm{B})\right\}
$$

ii.

$$
\begin{aligned}
\lambda^{\mu}(\mathrm{B}) & =\max \{\mu(\mathrm{y}) / \text { for all } \mathrm{y} \in \mathrm{B} \subseteq \mathrm{R}\} \\
& \leq \max \{\mu(\mathrm{xy}) / \text { for all } \mathrm{xy} \in \mathrm{AB} \subseteq \mathrm{R}\} \\
& \leq \lambda^{\mu}(\mathrm{AB}) \\
\lambda^{\mu}(\mathrm{AB}) & \geq \lambda^{\mu}(\mathrm{B})
\end{aligned}
$$

iii.

$$
\begin{aligned}
\lambda^{\mu}(\mathrm{A}) & =\max \{\mu(\mathrm{x}) / \text { for all } \mathrm{x} \in \mathrm{A} \subseteq \mathrm{R}\} \\
& \leq \max \{\mu(\mathrm{xy}) / \text { for all } \mathrm{xy} \in \mathrm{AB} \subseteq \mathrm{R}\} \\
& \leq \lambda^{\mu}(\mathrm{AB}) \\
\lambda^{\mu}(\mathrm{AB}) & \geq \lambda^{\mu}(\mathrm{A})
\end{aligned}
$$

Hence, $\lambda^{\mu}$ is a fuzzy HX ideal on $\vartheta$.

\subsection{Theorem}

\section{Proof}

Every fuzzy HX ideal $\lambda^{\mu}$ of a HX ring $\vartheta$ is a fuzzy HX k-ideal of HX ring $\vartheta$.

Let $\lambda^{\mu}$ be a fuzzy HX ideal of a HX ring $\vartheta$. Then,

$\min \left\{\lambda^{\mu}(\mathrm{A}+\mathrm{B}), \lambda^{\mu}(\mathrm{B})\right\}=\min \{\max \{\mu(\mathrm{x}+\mathrm{y}) /$ for all $\mathrm{x}+\mathrm{y} \in \mathrm{A}+\mathrm{B} \subseteq \mathrm{R}\}, \max \{\mu(\mathrm{y}) /$ for all $\mathrm{y} \in \mathrm{B} \subseteq \mathrm{R}\}\}$ $=\min \left\{\mu\left(\mathrm{x}_{0}+\mathrm{y}_{0}\right), \mu\left(\mathrm{y}_{0}\right)\right\}$

$\leq \mu\left(\mathrm{x}_{0}+\mathrm{y}_{0}-\mathrm{y}_{0}\right)$

$=\mu\left(\mathrm{x}_{0}\right)$

$\leq \max \{\mu(\mathrm{x}) /$ for all $\mathrm{x} \in \mathrm{A} \subseteq \mathrm{R}\}$

$$
\leq \lambda^{\mu}(\mathrm{A})
$$

Therefore, $\quad \lambda^{\mu}(\mathrm{A}) \quad \geq \min \left\{\lambda^{\mu}(\mathrm{A}+\mathrm{B}), \lambda^{\mu}(\mathrm{B})\right\}$

Hence, $\lambda^{\mu}$ is a fuzzy HX k-ideal of a HX ring $\vartheta$.

\subsection{Theorem}

Let $\mu$ and $\eta$ be two fuzzy ideals defined on R. Let $\lambda^{\mu}$ and $\gamma^{\eta}$ be any two fuzzy HX k-ideals of a HX ring $\vartheta$ then $\lambda^{\mu} \cap \gamma^{\eta}$ is also fuzzy HX k-ideal of a HX ring $\vartheta$.

Proof

Let $\mathrm{A}, \mathrm{B} \in \vartheta$

i. $\min \left\{\left(\lambda^{\mu} \cap \gamma^{\eta}\right)(\mathrm{A}),\left(\lambda^{\mu} \cap \gamma^{\eta}\right)(\mathrm{B})\right\}=\min \left\{\min \left\{\lambda^{\mu}(\mathrm{A}), \gamma^{\eta}(\mathrm{A})\right\}, \min \left\{\lambda^{\mu}(\mathrm{B}), \gamma^{\eta}(\mathrm{B})\right\}\right\}$ $\leq \min \left\{\min \left\{\lambda^{\mu}(\mathrm{A}), \lambda^{\mu}(\mathrm{B})\right\}, \min \left\{\gamma^{\eta}(\mathrm{A}), \gamma^{\eta}(\mathrm{B})\right\}\right\}$ $\leq \min \left\{\lambda^{\mu}(\mathrm{A}-\mathrm{B}), \gamma^{\eta}(\mathrm{A}-\mathrm{B})\right\}$

Since $\lambda^{\mu}$ and $\gamma^{\eta}$ are fuzzy HX k-ideals $\leq\left(\lambda^{\mu} \cap \gamma^{\eta}\right)(\mathrm{A}-\mathrm{B})$.

$\left.\left(\lambda^{\mu} \cap \gamma^{\eta}\right)(\mathrm{A}-\mathrm{B}) \quad \geq \min \left(\lambda^{\mu} \cap \gamma^{\eta}\right)(\mathrm{A}),\left(\lambda^{\mu} \cap \gamma^{\eta}\right)(\mathrm{B})\right\}$.

ii. $\quad\left(\lambda^{\mu} \cap \gamma^{\eta}\right)(\mathrm{A}) \quad=\min \left\{\lambda^{\mu}(\mathrm{A}), \gamma^{\eta}(\mathrm{A})\right\}$

$=\min \{\max \{\mu(\mathrm{x}) /$ for all $\mathrm{x} \in \mathrm{A} \subseteq \mathrm{R}\}, \max \{\eta(\mathrm{x}) /$ for all $\mathrm{x} \in \mathrm{A} \subseteq \mathrm{R}\}\}$

$\leq \min \{\max \{\mu(\mathrm{xy}) /$ for all $\mathrm{xy} \in \mathrm{AB} \subseteq \mathrm{R}\}, \max \{\eta(\mathrm{xy}) /$ for all $\mathrm{xy} \in \mathrm{AB} \subseteq \mathrm{R}\}\}$

$\leq \min \left\{\lambda^{\mu}(\mathrm{AB}), \gamma^{\eta}(\mathrm{AB})\right\}$

$\leq\left(\lambda^{\mu} \cap \gamma^{\eta}\right)(\mathrm{AB})$

$\left(\lambda^{\mu} \cap \gamma^{\eta}\right)(\mathrm{AB}) \quad \geq\left(\lambda^{\mu} \cap \gamma^{\eta}\right)(\mathrm{A})$.

iii. $\quad\left(\lambda^{\mu} \cap \gamma^{\eta}\right)(\mathrm{B}) \quad=\min \left\{\lambda^{\mu}(\mathrm{B}), \gamma^{\eta}(\mathrm{B})\right\}$

$=\min \{\max \{\mu(\mathrm{y}) /$ for all $\mathrm{y} \in \mathrm{B} \subseteq \mathrm{R}\}, \max \{\eta(\mathrm{y}) /$ for all $\mathrm{y} \in \mathrm{B} \subseteq \mathrm{R}\}\}$

$\leq \min \{\max \{\mu(\mathrm{xy}) /$ for all $\mathrm{xy} \in \mathrm{AB} \subseteq \mathrm{R}\}, \max \{\eta(\mathrm{xy}) /$ for all $\mathrm{xy} \in \mathrm{AB} \subseteq \mathrm{R}\}\}$

$\leq \min \left\{\lambda^{\mu}(\mathrm{AB}), \gamma^{\eta}(\mathrm{AB})\right\}$

$\leq\left(\lambda^{\mu} \cap \gamma^{\eta}\right)(\mathrm{AB})$

$\left(\lambda^{\mu} \cap \gamma^{\eta}\right)(\mathrm{AB}) \quad \geq\left(\lambda^{\mu} \cap \gamma^{\eta}\right)(\mathrm{B})$.

Hence, $\lambda^{\mu} \cap \gamma^{\eta}$ is fuzzy HX ideal of a HX ring $\vartheta$.

Now, $\min \left\{\left(\lambda^{\mu} \cap \gamma^{\eta}\right)(A+B),\left(\lambda^{\mu} \cap \gamma^{\eta}\right)(B)\right\}=\min \left\{\min \left\{\lambda^{\mu}(A+B), \gamma^{\eta}(A+B)\right\}, \min \left\{\lambda^{\mu}(B), \gamma^{\eta}(B)\right\}\right\}$ 


$$
\begin{aligned}
& \leq \min \left\{\min \left\{\lambda^{\mu}(\mathrm{A}+\mathrm{B}), \lambda^{\mu}(\mathrm{B})\right\}, \min \left\{\gamma^{\eta}(\mathrm{A}+\mathrm{B}), \gamma^{\eta}(\mathrm{B})\right\}\right\} \\
& \leq \min \left\{\lambda^{\mu}(\mathrm{A}+\mathrm{B}-\mathrm{B}), \gamma^{\eta}(\mathrm{A}+\mathrm{B}-\mathrm{B})\right\} \\
& \leq \min \left\{\lambda^{\mu}(\mathrm{A}), \gamma^{\eta}(\mathrm{A})\right\} \\
& =\left(\lambda^{\mu} \cap \gamma^{\eta}\right)(\mathrm{A})
\end{aligned}
$$

Hence, $\lambda^{\mu} \cap \gamma^{\eta}$ is a fuzzy HX k-ideal of a HX ring $\vartheta$.

\section{Remark}

Arbitrary intersection of a fuzzy HX k-ideal of a HX ring $\vartheta$ is also a fuzzy HX k-ideal of a HX ring $\vartheta$.

\section{Properties Of Level Subsets Of A Fuzzy HX K-Ideal Of A HX Ring}

In this section, we introduce the concept of level subsets of fuzzy HX k-ideal of a HX ring $\vartheta$ and discuss some of its properties.

\subsection{Definition}

Let $\lambda^{\mu}$ be a fuzzy HX k-ideal of a HX ring $\vartheta$. For any $t \in[0,1]$, we define the set $U\left(\lambda^{\mu} ; t\right)=\{A \in \vartheta /$ $\left.\lambda^{\mu}(A) \geq t\right\}$ is called an upper level subset or a level subset of $\lambda^{\mu}$.

\subsection{Theorem}

$\lambda^{\mu}$ be a fuzzy HX k-ideal of a HX ring $\vartheta$ if and only if $\mathrm{U}\left(\lambda^{\mu} ; \mathrm{t}\right)$ is either empty or a HX k-ideal of $\vartheta$ for all $t \in[0,1]$.

\section{Proof}

Let $\lambda^{\mu}$ be a fuzzy HX k-ideal of $\vartheta$.

Let $\mathrm{t} \in[0,1]$. Let $\mathrm{U}\left(\lambda^{\mu} ; \mathrm{t}\right) \neq \phi$.

For any $\mathrm{A}, \mathrm{B} \in \mathrm{U}\left(\lambda^{\mu} ; \mathrm{t}\right)$ and $\mathrm{C} \in \vartheta$, then $\lambda^{\mu}(\mathrm{A}) \geq \mathrm{t}$ and $\lambda^{\mu}(\mathrm{B}) \geq \mathrm{t}$.

Now, $\quad \lambda^{\mu}(A+C) \quad \geq \min \left\{\lambda^{\mu}(A), \lambda^{\mu}(C)\right\}$

$$
\lambda^{\mu}(\mathrm{AC}) \quad \geq \lambda^{\mu}(\mathrm{A}) \geq \mathrm{t} .
$$

Hence, $\mathrm{A}+\mathrm{C}, \mathrm{CB}$ and $\mathrm{AC} \in \mathrm{U}\left(\lambda^{\mu} ; \mathrm{t}\right)$.

Hence $\mathrm{U}\left(\lambda^{\mu} ; t\right)$ is an ideal of a HX ring $\vartheta$.

For any $\mathrm{A} \in \mathrm{U}\left(\lambda^{\mu} ; \mathrm{t}\right)$ and $\mathrm{C} \in \vartheta$ then $\mathrm{A}+\mathrm{C}$ or $\mathrm{C}+\mathrm{A} \in \mathrm{U}\left(\lambda^{\mu} ; \mathrm{t}\right)$,

then $\quad \lambda^{\mu}(\mathrm{A}) \geq \mathrm{t}$ and $\lambda^{\mu}(\mathrm{C}+\mathrm{A}) \geq \mathrm{t}$ or $\lambda^{\mu}(\mathrm{A}+\mathrm{C}) \geq \mathrm{t}$.

Now, $\quad \lambda^{\mu}(\mathrm{C}) \geq \min \left\{\lambda^{\mu}(\mathrm{C}+\mathrm{A}), \lambda^{\mu}(\mathrm{A})\right\} \geq \mathrm{t}$. Hence $\mathrm{C} \in \mathrm{U}\left(\lambda^{\mu} ; \mathrm{t}\right)$.

Hence $\mathrm{U}\left(\lambda^{\mu} ; \mathrm{t}\right)$ is a HX k-ideal of a HX ring $\vartheta$.

Conversely, let $\mathrm{U}\left(\lambda^{\mu} ; \mathrm{t}\right)$ is HX k-ideal of a HX ring $\vartheta$.

For any $\mathrm{A}, \mathrm{B} \in \mathrm{U}\left(\lambda^{\mu} ; \mathrm{t}\right)$ and $\mathrm{C} \in \vartheta$, then $\mathrm{A}+\mathrm{C}, \mathrm{CB}$ and $\mathrm{AC} \in \mathrm{U}\left(\lambda^{\mu} ; \mathrm{t}\right)$ and

For any $\mathrm{A} \in \mathrm{U}\left(\lambda^{\mu} ; \mathrm{t}\right), \mathrm{C} \in \vartheta$ and $\mathrm{A}+\mathrm{C}$ or $\mathrm{C}+\mathrm{A} \in \mathrm{U}\left(\lambda^{\mu} ; \mathrm{t}\right)$ then $\mathrm{C} \in \mathrm{U}\left(\lambda^{\mu} ; \mathrm{t}\right)$.

For any $A, B \in \vartheta$, then $A, B \in U\left(\lambda^{\mu} ; t\right)$ for some $t \in[0,1]$ and

Therefore, $\mathrm{A}+\mathrm{B}, \mathrm{A}-\mathrm{B}$ and $\mathrm{AB} \in \mathrm{U}\left(\lambda^{\mu} ; \mathrm{t}\right)$.

Let $\lambda^{\mu}(A)=t_{1}, \lambda^{\mu}(B)=t_{2}, \lambda^{\mu}(A-B)=t_{3}$ and $\lambda^{\mu}(A B)=t_{4}$ such that $0 \leq t_{1} \leq t_{2} \leq t_{3} \leq t_{4} \leq t_{\text {t }}$

Now, $\lambda^{\mu}(\mathrm{A}-\mathrm{B}) \quad=\mathrm{t}_{3}$

$\geq \mathrm{t}_{1}$

That is, $\begin{aligned} \lambda^{\mu}(\mathrm{A}-\mathrm{B}) & =\min \left\{\lambda^{\mu}(\mathrm{A}), \lambda^{\mu}(\mathrm{B})\right\} . \\ & \geq \min \left\{\lambda^{\mu}(\mathrm{A}), \lambda^{\mu}(\mathrm{B})\right\} .\end{aligned}$

$\lambda^{\mu}(\mathrm{AB}) \quad=\mathrm{t}_{4}$

$\begin{aligned} \lambda^{\mu}(\mathrm{AB}) & \geq \mathrm{t}_{2} \\ & \geq \lambda^{\mu}(\mathrm{B}) .\end{aligned}$

$\begin{aligned} \lambda^{\mu}(\mathrm{AB}) & \geq \mathrm{t}_{1} \\ & \geq \lambda^{\mu}(\mathrm{A}) .\end{aligned}$

Hence, $\lambda^{\mu}$ is a fuzzy HX ideal of a HX ring $\vartheta$.

By Theorem 3.6, $\lambda^{\mu}$ is a fuzzy HX k-ideal of a HX ring $\vartheta$.

\subsection{Definition}

Let $\lambda^{\mu}$ be a fuzzy HX k-ideal of a HX ring $\vartheta$. Then, the $\mathrm{HX}$ k-ideals $\mathrm{U}\left(\lambda^{\mu} ; \mathrm{t}\right)$ for $\mathrm{t} \in[0,1]$ and $\mathrm{t} \leq \lambda^{\mu}(\mathrm{Q})$, are called level HX k-ideals of $\lambda^{\mu}$. 


\subsection{Theorem}

Let $\lambda^{\mu}$ be a fuzzy HX k-ideal of a HX ring $\vartheta$. If two level HX k-ideals $U\left(\lambda^{\mu} ; t_{1}\right), U\left(\lambda^{\mu} ; t_{2}\right)$ for, $\mathrm{t}_{1}, \mathrm{t}_{2} \in[0,1]$ and $\mathrm{t}_{1}, \mathrm{t}_{2} \leq \lambda^{\mu}(\mathrm{Q})$ with $\mathrm{t}_{1}<\mathrm{t}_{2}$ of $\lambda^{\mu}$ are equal then there is no $\mathrm{A}$ in $\vartheta$ such that $\mathrm{t}_{1} \leq \lambda^{\mu}(\mathrm{A})<\mathrm{t}_{2}$.

\section{Proof}

Let $\mathrm{U}\left(\lambda^{\mu} ; \mathrm{t}_{1}\right)=\mathrm{U}\left(\lambda^{\mu} ; \mathrm{t}_{2}\right)$. Suppose there exists $\mathrm{A} \in \vartheta$ such that $\mathrm{t}_{1} \leq \lambda^{\mu}(\mathrm{A})<\mathrm{t}_{2}$, then $\mathrm{U}\left(\lambda^{\mu} ; \mathrm{t}_{2}\right) \subseteq$ $\mathrm{U}\left(\lambda^{\mu} ; \mathrm{t}_{1}\right)$. Then $\mathrm{A} \in \mathrm{U}\left(\lambda^{\mu} ; \mathrm{t}_{1}\right)$, but $\mathrm{A} \notin \mathrm{U}\left(\lambda^{\mu} ; \mathrm{t}_{2}\right)$, which contradicts the assumption that, $\mathrm{U}\left(\lambda^{\mu} ; \mathrm{t}_{1}\right)=\mathrm{U}\left(\lambda^{\mu} ; \mathrm{t}_{2}\right)$. Hence there is no A in $\vartheta$ such that $t_{1} \leq \lambda^{\mu}(A)<t_{2}$.

Conversely, suppose that there is no $A$ in $\vartheta$ such that $t_{1} \leq \lambda^{\mu}(A)<t_{2}$, Then by definition $U\left(\lambda^{\mu} ; t_{2}\right)$ $\subseteq \mathrm{U}\left(\lambda^{\mu} ; \mathrm{t}_{1}\right)$. Let $\mathrm{A} \in \mathrm{U}\left(\lambda^{\mu} ; \mathrm{t}_{1}\right)$ and there is no $\mathrm{A}$ in $\vartheta$ such that $\mathrm{t}_{1} \leq \lambda^{\mu}(\mathrm{A})<\mathrm{t}_{2}$.

Therefore, $\mathrm{A} \in \mathrm{U}\left(\lambda^{\mu} ; \mathrm{t}_{2}\right)$ and $\mathrm{U}\left(\lambda^{\mu} ; \mathrm{t}_{1}\right) \subseteq \mathrm{U}\left(\lambda^{\mu} ; \mathrm{t}_{2}\right)$.

Hence, $\mathrm{U}\left(\lambda^{\mu} ; \mathrm{t}_{1}\right)=\mathrm{U}\left(\lambda^{\mu} ; \mathrm{t}_{2}\right)$.

\subsection{Theorem}

A fuzzy subset $\lambda^{\mu}$ of $\vartheta$ is a fuzzy HX k-ideal of a HX ring $\vartheta$ if and only if the level HX k-ideals $\mathrm{U}\left(\lambda^{\mu} ; \mathrm{t}\right), \mathrm{t} \in$ Image $\lambda^{\mu}$, are HX k-ideals of $\vartheta$.

Proof

It is clear.

\subsection{Theorem}

Any HX k-ideal $\mathrm{H}$ of a HX ring $\vartheta$ can be realized as a level HX k-ideal of some fuzzy HX left k-ideal of $\vartheta$.

Proof

Let $\lambda^{\mu}$ be a fuzzy subset and $A \in \vartheta$. Define,

Let $\mathrm{A}, \mathrm{B} \in \vartheta$.

$$
\lambda^{\mu}(A)= \begin{cases}t & \text { if } A \in H, \text { where } t \in(0,1] \\ 0 & \text { if } A \notin H,\end{cases}
$$

i. $\quad$ Suppose $A, B \in H$, then $A+B \in H, A-B \in H$ and $A B \in H$.

$\lambda^{\mu}(\mathrm{A})=\mathrm{t}, \lambda^{\mu}(\mathrm{B})=\mathrm{t}, \lambda^{\mu}(\mathrm{A}+\mathrm{B})=\mathrm{t}, \lambda^{\mu}(\mathrm{A}-\mathrm{B})=\mathrm{t}$ and $\lambda^{\mu}(\mathrm{AB})=\mathrm{t}$.

Hence $\quad \lambda^{\mu}(\mathrm{A}-\mathrm{B}) \geq \min \left\{\lambda^{\mu}(\mathrm{A}), \lambda^{\mu}(\mathrm{B})\right\}$,

$\lambda^{\mu}(\mathrm{AB}) \geq \lambda^{\mu}(\mathrm{B}), \quad$ and

$\lambda^{\mu}(\mathrm{A}) \geq \min \left\{\lambda^{\mu}(\mathrm{A}+\mathrm{B}), \lambda^{\mu}(\mathrm{B})\right\}$.

ii. $\quad$ Suppose $A \in H$ and $B \notin H$, then $A+B \notin H, A-B \notin H$ and $A B \notin H$.

$\lambda^{\mu}(\mathrm{A})=\mathrm{t}, \lambda^{\mu}(\mathrm{B})=0, \lambda^{\mu}(\mathrm{A}+\mathrm{B})=0, \lambda^{\mu}(\mathrm{A}-\mathrm{B})=0$ and $\lambda^{\mu}(\mathrm{AB})=0$.

Hence $\quad \lambda^{\mu}(\mathrm{A}-\mathrm{B}) \geq \min \left\{\lambda^{\mu}(\mathrm{A}), \lambda^{\mu}(\mathrm{B})\right\}$,

$\lambda^{\mu}(\mathrm{AB}) \geq \lambda^{\mu}(\mathrm{B})$, and

$\lambda^{\mu}(\mathrm{A}) \geq \min \left\{\lambda^{\mu}(\mathrm{A}+\mathrm{B}), \lambda^{\mu}(\mathrm{B})\right\}$.

iii. $\quad$ Suppose $A, B \notin H$, then $A+B \in H$ or $A+B \notin H$ and $A B \in H$ or $A B \notin H$.

$\lambda^{\mu}(\mathrm{A})=0, \lambda^{\mu}(\mathrm{B})=0, \lambda^{\mu}(\mathrm{A}+\mathrm{B})=\mathrm{t}$ or $0, \lambda^{\mu}(\mathrm{A}-\mathrm{B})=\mathrm{t}$ or 0 and $\lambda^{\mu}(\mathrm{AB})=\mathrm{t}$ or 0 .

Hence $\quad \lambda^{\mu}(\mathrm{A}-\mathrm{B}) \geq \min \left\{\lambda^{\mu}(\mathrm{A}), \lambda^{\mu}(\mathrm{B})\right\}$,

$\lambda^{\mu}(\mathrm{AB}) \geq \lambda^{\mu}(\mathrm{B})$, and

$\lambda^{\mu}(\mathrm{A}) \geq \min \left\{\lambda^{\mu}(\mathrm{A}+\mathrm{B}), \lambda^{\mu}(\mathrm{B})\right\}$.

Thus in all cases, $\lambda^{\mu}$ is a fuzzy HX left k-ideal of $\vartheta$. For this fuzzy HX left k-ideal, $U\left(\lambda^{\mu} ; \mathrm{t}\right)=\mathrm{H}$.

\section{Remark}

As a consequence of the Theorem 4.4 and 4.5, the level HX k-ideals of a fuzzy HX k-ideal $\lambda^{\mu}$ of a HX ring $\vartheta$ form a chain. Since $\lambda^{\mu}(Q) \geq \lambda^{\mu}(A)$ for all $A$ in $\vartheta$ and therefore $U\left(\lambda^{\mu} ; t_{0}\right)$, where $\lambda^{\mu}(Q)=t_{0}$ is the smallest and we have the chain $:\{\mathrm{Q}\}=\mathrm{U}\left(\lambda^{\mu} ; \mathrm{t}_{0}\right) \subset \mathrm{U}\left(\lambda^{\mu} ; \mathrm{t}_{1}\right) \subset \mathrm{U}\left(\lambda^{\mu} ; \mathrm{t}_{2}\right) \subset \ldots \subset \mathrm{U}\left(\lambda^{\mu} ; \mathrm{t}_{\mathrm{n}}\right)=\vartheta$, where $\mathrm{t}_{0}>\mathrm{t}_{1}>\mathrm{t}_{2}>\ldots \ldots>\mathrm{t}_{\mathrm{n}}$

\section{Conclusion}

In fuzzy algebraic system fuzzy ideals with special properties plays an important role. In this paper we defined an induced fuzzy k-ideal of a HX ring where k-ideals provides an important tool to construct quotient rings. Hence the level HX k-ideals of a HX ring $\vartheta$ form a chain and some properties were discussed, which will give a new horizon in the further study. 


\section{Acknowledgements}

The authors would like to express their sincere thanks to all our friends for their help to make this paper a successful one.

\section{References}

[1] Bing-Xue Yao And Yubin-Zhong, The Construction Of Power Ring, Fuzzy Information And Engineering (ICFIE),ASC 40,Pp.181187,2007

[2] Bing-Xue Yao And Yubin-Zhong, Upgrade Of Algebraic Structure Of Ring, Fuzzy Information And Engineering (2009)2:219-228.

[3] Dheena.P And Mohanraaj.G, T- Fuzzy Ideals In Rings ,International Journal Of Computational Cognition, Volume 9, No.2, 98101, June 2011 .

[4] Dheena.P And Mohanraaj.G, On Intuitionistic Fuzzy K Ideals Of Semirings , International Journal Of Computational Cognition, Volume 9, No.2, 45-50, June 2011

[5] Dutta T. K. And Biswas . B.K., Fuzzy K-Ideals Of Semirings,Bull, Calcutta Math.Soc.,87,91-96

[6] Ghosh.S, Fuzzy K-Ideals Of Semiring, Fuzzy Sets And Systems, 95,103-108,1998.

[7] Jun Y. B., Neggers. J. And Kim. H. S, On L-Fuzzy Ideals In Semirings I, Czech. Math. J. 48 (1998), 669-675.

[8] Kim.C.B And Park.M , K-Fuzzy Ideals In Semirings,Fuzzy Sets And Systems, 81, 281-286, 1996.

[9] Li Hong Xing, HX Group, BUSEFAL,33(4), 31-37, October 1987.

[10] Liu. W.J., Fuzzy Invariant Subgroups And Fuzzy Ideals, Fuzzy Sets And Systems,8:133-139.

[11] Li Hong Xing, HX Ring, BUSEFAL ,34(1) 3-8,January 1988.

[12] Mashinchi.M And Zahedi.M.M, On Fuzzy Ideals Of A Ring,J.Sci.I.R.Iran,1(3),208- 210(1990)

[13] Mukherjee.T.K \& Sen.M.K., On Fuzzy Ideals In Rings, Fuzzy Sets And Systems, 21, 99- 104,1987.

[14] Rosenfeld. A., Fuzzy Groups,J.Math.Anal.,35(1971),512-517.

[15] Seung Il Baik And Hee Sik Kim , On Fuzzy K-Ideals In Semirings, Kangweon - Kyungki Math.Jour.8(2000),No.2.Pp,147-154

[16] Zadeh.L.A., Fuzzy Sets, Information And Control,8,338-353. 